\title{
An elementary operational calculus
}

\section{DRAGU ATANASIU and PIOTR MIKUSIŃSKI}

\section{Introduction}

One of the standard methods presented in an introductory course on differential equations is the Laplace transform method. While it has some attractive features, it presents difficulties, like the unnatural restriction to functions of exponential growth. Moreover, a rigorous treatment quickly leads to non-elementary mathematics. In the 1950s Jan Mikusiński [1] proposed an operational calculus that offered certain advantages over the Laplace transform. It has not replaced the Laplace transform method in elementary courses, possibly because it still required a high level of mathematical sophistication. Even the simplified operational calculus proposed by Kosaku Yosida and Shuichi Okamoto in [2] (see also [3]) has not succeeded in becoming a standard textbook method.

In this note we propose a simple alternative to the Laplace transform method for an elementary course in differential equations. The method can be applied to a larger class of functions, since growth conditions play no role.

\section{A simple approach}

All functions considered in this section are continuous functions defined on $[0, \infty]$. The main tool is the convolution of functions:

$$
f * g(t)=\int_{0}^{t} f(s) g(t-s) d s .
$$

We shall make use of basic algebraic properties of the convolution: $f * g=g * f,(f * g) * h=f *(g * h), a(f * g)=(a f) * g=f *(a g)$, $f *(g+h)=f * g+f * h$, where $f, g$ and $h$ are arbitrary functions, and $a$ is an arbitrary number. All these properties follow directly from the definition by elementary properties of integration and should be easy exercises for students.

In our approach the function $\omega(t)=t$ plays a special role. First, we observe that $\omega * f=0$ implies $f=0$. Since

$$
\omega * f(t)=\int_{0}^{t} f(s)(t-s) d s=t \int_{0}^{t} f(s) d s-\int_{0}^{t} s f(s) d s
$$

by the Fundamental Theorem of Calculus we conclude that $\omega * f$ is differentiable and we have

$$
(\omega * f)^{\prime}(t)=\int_{0}^{t} f(s) d s .
$$

If $\omega * f=0$, then $\int_{0}^{t} f(s) d s=0$ for every $t \in[0, \infty)$, and hence $f=0$. This gives us the first important property:

$$
\omega * f=\omega * g \text { implies } f=g .
$$


To obtain the next formula we assume that $y$ is continuously differentiable and consider the convolution $\omega * y^{\prime}$ :

$$
\begin{aligned}
\omega * y^{\prime}(t) & =\int_{0}^{t}(t-s) y^{\prime}(s) d s \\
& =t \int_{0}^{t} y^{\prime}(s) d s-\int_{0}^{t} s y^{\prime}(s) d s \\
& =t(y(t)-y(0))-t y(t)+\int_{0}^{t} y(s) d s \\
& =\int_{0}^{t} y(s) d s-t y(0) .
\end{aligned}
$$

This gives us the second important property:

$$
\omega * y^{\prime}=\omega^{\prime} * y-y(0) \omega .
$$

As a direct consequence of the above we obtain another useful property:

$$
\left(\omega^{\prime}-a \omega\right) * E_{a}=\omega
$$

where $E_{a}(t)=e^{a t}$.

Properties (1), (2) and (3) are sufficient for effectively solving any equation of the form $y^{\prime}-a y=f$, where $f$ is an arbitrary continuous function on $[0, \infty)$. Moreover, the method gives us an elementary proof of existence and uniqueness of solutions for this class of equations. To deal with linear equations of higher order it is necessary to generalise the second property. We replace $y$ by $y^{\prime}$ in (2) and convolve both sides with $\omega$ to obtain

$$
\omega * \omega * y^{\prime \prime}=\omega * \omega^{\prime} * y^{\prime}-y^{\prime}(0) \omega * \omega .
$$

Then we use the property $\omega * \omega^{\prime}=\omega^{\prime} * \omega$ and apply (2) again to derive the following formula

$$
\omega * \omega * y^{\prime \prime}=\omega^{\prime} * \omega^{\prime} * y-y(0) \omega^{\prime} * \omega-y^{\prime}(0) \omega * \omega
$$

which holds when $y$ is twice continuously differentiable.

Now we show how these ideas can be used in practice. Consider the equation

$$
y^{\prime \prime}-5 y^{\prime}+6 y=f, y(0)=a, y^{\prime}(0)=b
$$

where $f$ is an arbitrary continuous function defined on $[0, \infty)$ and $a$ and $b$ are arbitrary real numbers.

Since it is a second order equation, we convolve both sides of the equation with $\omega * \omega$ and then use properties (2) and (4). After regrouping we obtain

$$
\left(\omega^{\prime}-2 \omega\right) *\left(\omega^{\prime}-3 \omega\right) * y-a \omega^{\prime} * \omega+(5 a-b) \omega * \omega=\omega * \omega * f .
$$

The presence of factors $\left(\omega^{\prime}-2 \omega\right)$ and $\left(\omega^{\prime}-3 \omega\right)$ suggests convolving with $E_{2} * E_{3}$ and then using (3). Thus we obtain

$$
\omega * \omega * y-a \omega^{\prime} * \omega * E_{2} * E_{3}+(5 a-b) \omega * \omega * E_{2} * E_{3}=\omega * \omega * E_{2} * E_{3} * f \text {. }
$$


Note that for $\alpha \neq \beta$ we have

$$
E_{\alpha} * E_{\beta}=\frac{1}{\alpha-\beta}\left(E_{\alpha}-E_{\beta}\right),
$$

which can easily be derived from the definition of convolution:

$$
\begin{aligned}
E_{\alpha} * E_{\beta}(t) & =\int_{0}^{t} e^{\alpha(t-x)} e^{\beta x} d x=e^{\alpha t} \int_{0}^{t} e^{(\beta-\alpha) x} d x \\
& =\frac{1}{\beta-\alpha}\left(e^{\beta t}-e^{\alpha t}\right)=\frac{1}{\alpha-\beta}\left(e^{\alpha t}-e^{\beta t}\right) .
\end{aligned}
$$

By applying (7) and (2) we get

$$
-a \omega^{\prime} * \omega * E_{2} * E_{3}=a\left(2 E_{2}-3 E_{3}\right) * \omega * \omega .
$$

Using (7) and (8) we can rewrite (6) as

$$
\omega * \omega * y=\omega * \omega *\left((3 a-b) E_{2}+(-2 a+b) E_{3}+\left(E_{3}-E_{2}\right) * f\right) .
$$

Finally, after using (1), we obtain the solution

$$
y=(3 a-b) E_{2}+(-2 a+b) E_{3}+\left(E_{3}-E_{2}\right) * f .
$$

Note that the presented method can be used even if $f$ is not Laplace transformable, for example if $f(t)=e^{t^{2}}$.

The definition of convolution gives us

$$
E_{\alpha} * E_{\alpha}=\omega E_{\alpha} .
$$

If $\alpha$ and $\beta$ are conjugate complex numbers, $\alpha=a+b i$ and $\beta=a-b i$ $(b \neq 0)$, then using $(7)$ we obtain

$$
E_{\alpha} * E_{\beta}=\frac{1}{b} E_{a} S_{b}
$$

where $S_{b}(t)=\sin b t$.

Using formulae (10) and (11) we can solve other types of second order linear equations. It is clear that the method can be used to solve equations of higher order.

Now we consider the equation (5) where $f$ is a specific function, namely $f=\omega E_{2}$, that is $f(t)=t e^{2 t}$. To use (9) we need to find

$$
\left(E_{3}-E_{2}\right) *\left(\omega E_{2}\right)=E_{3} *\left(\omega E_{2}\right)-E_{2} *\left(\omega E_{2}\right) .
$$

For $\alpha \neq \beta$, using (7) and (10), we calculate

$$
\begin{aligned}
E_{\alpha} *\left(\omega E_{\beta}\right) & =E_{\alpha} *\left(E_{\beta} * E_{\beta}\right) \\
& =\left(E_{\alpha} * E_{\beta}\right) * E_{\beta} \\
& =\frac{1}{\alpha-\beta}\left(E_{\alpha}-E_{\beta}\right) * E_{\beta}
\end{aligned}
$$




$$
\begin{aligned}
& =\frac{1}{\alpha-\beta}\left(E_{\alpha} * E_{\beta}-E_{\beta} * E_{\beta}\right) \\
& =\frac{1}{(\alpha-\beta)^{2}}\left(E_{\alpha}-E_{\beta}\right)-\frac{1}{\alpha-\beta} \omega E_{\beta} .
\end{aligned}
$$

Since

$$
\left(E_{\alpha} * \omega E_{\alpha}\right)(t)=\int_{0}^{t} e^{\alpha(t-x)} x e^{\alpha x} d x=e^{\alpha t} \int_{0}^{t} x d x=\frac{1}{2} t^{2} e^{\alpha t}
$$

we get

$$
E_{\alpha} * \omega E_{\alpha}=\frac{1}{2} \omega^{2} E_{\alpha}
$$

Hence

$$
\left(E_{3}-E_{2}\right) *\left(\omega E_{2}\right)=E_{3}-E_{2}-\omega E_{2}-\frac{1}{2} \omega^{2} E_{2}=E_{3}-\left(1+\omega+\frac{1}{2} \omega^{2}\right) E_{2} .
$$

Consequently the solution of the equation

$$
y^{\prime \prime}-5 y^{\prime}+6 y=t e^{2 t}, y(0)=a, y^{\prime}(0)=b
$$

is

$$
y(t)=(3 a-b) e^{2 t}+(-2 a+b) e^{3 t}+e^{3 t}-\left(1+t+\frac{1}{2} t^{2}\right) e^{2 t} .
$$

Note that $3 a-b$ and $-2 a+b$ could be arbitrary constants and hence $(3 a-b) e^{2 t}+(-2 a+b) e^{3 t}$ can be written in the standard form of solution of the homogeneous equation $y^{\prime \prime}-5 y^{\prime}+6 y=0$. It is the solution of this homogeneous equation with $y(0)=a$ and $y^{\prime}(0)=b$. On the other hand, $e^{3 t}-\left(1+t+\frac{1}{2} t^{2}\right) e^{2 t}$ is the solution of the non-homogeneous equation $y^{\prime \prime}-5 y^{\prime}+6 y=t e^{2 t}$ with $y(0)=0$ and $y^{\prime}(0)=0$.

Another typical example of equation (5) is an equation with a function $f$ where sine or cosine is involved. Consider, for example, the equation $y^{\prime \prime}-5 y^{\prime}+6 y=\cos t$ with $y(0)=a$ and $y^{\prime}(0)=b$. To facilitate calculations we denote $S(t)=\sin t$ and $C(t)=\cos t$ and use the identities

$$
C=\frac{E_{i}+E_{-i}}{2} \quad \text { and } \quad S=\frac{E_{i}+E_{-i}}{2 i} .
$$

Finding the solution requires calculating $\left(E_{3}-E_{2}\right) * C$ :

$$
\begin{aligned}
\left(E_{3}-E_{2}\right) * C & =E_{3} *\left(\frac{E_{i}+E_{-i}}{2}\right)-E_{2} *\left(\frac{E_{i}+E_{-i}}{2}\right) \\
& =\mathscr{R}\left(E_{3} * E_{i}\right)-\mathscr{R}\left(E_{2} * E_{i}\right) \\
& =\mathscr{R}\left(\frac{1}{3-i}\left(E_{3}-E_{i}\right)\right)-\mathscr{R}\left(\frac{1}{2-i}\left(E_{2}-E_{i}\right)\right) \\
& =\frac{3}{10} E_{3}-\frac{2}{5} E_{2}+\frac{1}{10} C-\frac{1}{10} S .
\end{aligned}
$$

where $\mathscr{R}$ means the real part. 
Consequently, the solution of the equation

$$
y^{\prime \prime}-5 y^{\prime}+6 y=\cos t, \quad y(0)=a, \quad y^{\prime}(0)=b
$$

is

$$
y(t)=(3 a-b) e^{2 t}+(-2 a+b) e^{3 t}+\frac{3}{10} e^{3 t}-\frac{2}{5} e^{2 t}+\frac{1}{10} \cos t-\frac{1}{10} \sin t .
$$

\section{A more advanced approach}

In the formula

$$
\omega^{\prime} * y=\omega * y^{\prime}+y(0) \omega
$$

obtained in the previous section, the term $\omega * y^{\prime}$ can be identified with the derivative of $y$. If $y$ is considered as a function defined on the real line such that $y(t)=0$ for $t<0$, then the term $y(0) \omega$ stores the information about the jump of $y$ at 0 . This means that $\omega^{\prime} * y$ is more than the derivative of $y$. We will call it the generalised derivative of $y$.

Consider the equation

$$
\omega^{\prime} * y-a(\omega * y)=b \omega,
$$

where $a$ and $b$ are real numbers. We rewrite the equation as

$$
\left(\omega^{\prime}-a \omega\right) * y=b \omega,
$$

convolve both sides with $E_{a}$ and use (3) to obtain

$$
\omega * y=\omega * b E_{a} .
$$

Hence, by (1),

$$
y=b E_{a} .
$$

As mentioned above, this solution should be interpreted as

$$
y(t)=\left\{\begin{array}{cl}
b e^{a t} & \text { if } t \geqslant 0, \\
0 & \text { if } t<0 .
\end{array}\right.
$$

When $a=0$ and $b=1$ the equation becomes

$$
\omega^{\prime} * y=\omega
$$

and the solution is

$$
y(t)= \begin{cases}1 & \text { if } t \geqslant 0, \\ 0 & \text { if } t<0 .\end{cases}
$$

This is the Heaviside function.

In a standard differential equations course, the equation (12) is written as

$$
y^{\prime}-a y=b \delta
$$


In order to give an elementary and rigorous method for solving equations of type (13) we have identified $y$ with $\omega * y, y^{\prime}$ with $\omega^{\prime} * y$, and $\delta$ with $\omega$.

We observe that the equality $\omega=\omega * f$ is not possible for any nonzero function $f$. This implies that in our identification $f \rightarrow \omega * f$, there is no function $g$ such that $g \rightarrow \omega$.

For differential equations of higher order, the method has to be modified as in Section 2. For example, for second order equations, we use the identification $y \rightarrow \omega * \omega * y$.

Acknowledgement: The authors would like to thank the referee whose comments helped us improve this note.

\section{References}

1. Jan Mikusiński, Operational Calculus, Pergamon Press (1959).

2. Kosaku Yosida and Shuichi Okamoto, A note on Mikusiński's operational calculus, Proc. Japan Acad. Ser. A Math. Sci., 56 (1980), pp. 1-3.

3. Kosaku Yosida, Operational calculus; a theory of hyperfunctions, Springer-Verlag (1984).

doi: $10.1017 / \mathrm{mag} .2014 .7$

DRAGU ATANASIU

University of Borås, Borås, Sweden

e-mail: dragu.atanasiu@hb.se

PIOTR MIKUSIŃSKI

Department of Mathematics, University of Central Florida, Orlando,

FL 32816-1364, USA

e-mail: piotr.mikusinski@ucf.edu 